\title{
Epigenetic status of argininosuccinate synthetase and argininosuccinate lyase modulates autophagy and cell death in glioblastoma
}

\author{
N Syed ${ }^{*, 1}$, J Langer ${ }^{1}, \mathrm{~K}$ Janczar ${ }^{1}$, P Singh ${ }^{1}$, C Lo Nigro ${ }^{2}$, L Lattanzio ${ }^{2}$, HM Coley $^{3}$, E Hatzimichael ${ }^{4}$, J Bomalaski ${ }^{5}$, P Szlosarek ${ }^{6}$, \\ M Awad ${ }^{7}, \mathrm{~K} \mathrm{O}^{\prime} \mathrm{Neil}^{7}$, F Roncaroli ${ }^{1}$ and T Crook $^{8}$
}

Arginine deprivation, either by nutritional starvation or exposure to ADI-PEG20, induces adaptive transcriptional upregulation of ASS1 and ASL in glioblastoma multiforme ex vivo cultures and cell lines. This adaptive transcriptional upregulation is blocked by neoplasia-specific $\mathrm{CpG}$ island methylation in either gene, causing arginine auxotrophy and cell death. In cells with methylated ASS1 or ASL CpG islands, ADI-PEG20 initially induces a protective autophagic response, but abrogation of this by chloroquine accelerates and potentiates cytotoxicity. Concomitant methylation in the CpG islands of both ASS1 and ASL, observed in a subset of cases, confers hypersensitivity to ADI-PEG20. Cancer stem cells positive for CD133 and methylation in the ASL CpG island retain sensitivity to ADI-PEG20. Our results show for the first time that epigenetic changes occur in both of the two key genes of arginine biosynthesis in human cancer and confer sensitivity to therapeutic arginine deprivation. We demonstrate that methylation status of the CpG islands, rather than expression levels per se of the genes, predicts sensitivity to arginine deprivation. Our results suggest a novel therapeutic strategy for this invariably fatal central nervous system neoplasm for which we have identified robust biomarkers and which overcomes the limitations to conventional chemotherapy imposed by the blood/ brain barrier.

Cell Death and Disease (2013) 4, e458; doi:10.1038/cddis.2012.197; published online 17 January 2013

Subject Category: Cancer

Glioblastoma multiforme (GBM) is the most common primary human malignant brain tumour and is among the most lethal of all cancers. ${ }^{1}$ Despite advances in surgical management and radiotherapy, and development of temozolomide, the median survival for patients is $12-16$ months. ${ }^{2,3}$ The unfavourable prognosis in this disease is strongly correlated to the intrinsic apoptosis resistance of GBM. ${ }^{4,5}$ Disease recurrence after treatment is mechanistically linked to the presence of chemo-/ radio-resistant $\mathrm{CD} 133^{+}$cancer stem cells (CSCs). ${ }^{6} \mathrm{~A}$ further barrier to effective therapy of GBM is the presence of the blood/brain barrier, which limits access of chemotherapy to the central nervous system. These considerations clearly underline the urgent need for novel therapeutic approaches. GBM cells are less sensitive to apoptosis, ${ }^{4}$ yet less resistant to therapies that induce autophagy. ${ }^{7-9}$ Temozolomide, the most active agent in treating GBM, exerts cytotoxicity by inducing autophagic cell death. ${ }^{10}$ Autophagy is a non-apoptotic route of programmed cell death involving the formation of large double-membrane autophagic vacuoles termed autophagosomes $^{11,12}$ to degrade and recycle long-lived organelles and proteins by merging with lysosomes to form autolysosomes. Autophagy has a critical role in cellular survival during periods of starvation, but when stress conditions are excessive, autophagy becomes a cellular suicide pathway. As such, autophagy is initially an adaptive response to ensure survival but beyond a certain threshold may mediate a form of noncaspase-mediated cell death.

Tumour cells have a high requirement for arginine. This semi-essential amino acid is synthesized from citrulline via the urea cycle enzymes argininosuccinate synthetase (ASS) and argininosuccinate lyase (ASL). ${ }^{13}$ In a subset of human cancers, ASS expression is lost rendering them highly sensitive to deprivation of arginine by pegylated arginine deiminase (ADI-PEG20). ${ }^{14-21}$ ADI-PEG20 induces autophagic cell death in melanoma and in prostate carcinoma. ${ }^{18,22}$ ASS and ASL are tightly coupled and upregulated

\footnotetext{
${ }^{1}$ John Fulcher Neuro-oncology laboratory, Division of Brain Sciences, Faculty of Medicine, Imperial College London, London W6 8RP, UK; ${ }^{2}$ Laboratory of Cancer Genetics and Translational Oncology, S. Croce General Hospital, Cuneo, Italy; ${ }^{3}$ Faculty of Health and Medical Sciences, University of Surrey, Surrey GU2 7XH, UK; ${ }^{4}$ Hematology Research Laboratory, University Hospital of loannina, loannina, Greece; ${ }^{5}$ Polaris Pharmaceuticals Inc., 9373 Towne Centre Drive, Suite 150, San Diego, CA, USA; ${ }^{6}$ Barts Cancer Institute, Queen Mary University of London, Centre for Molecular Oncology, Charterhouse Square, London, UK; ${ }^{7}$ Department of Neurosurgery, Charing Cross Hospital, London W6 8RF, UK and ${ }^{8}$ Division of Cancer Research, Medical Research Institute, University of Dundee, Ninewells Hospital and Medical School, Dundee DD1 9SY, UK

${ }^{*}$ Corresponding author: N Syed, John Fulcher Neuro-oncology laboratory, Division of Brain Sciences, Faculty of Medicine, Imperial College London, London W6 8RP, UK. Tel: + 440208846 7029; Fax: + 440208846 7794; E-mail: n.syed@imperial.ac.uk

Keywords: glioblastoma; arginine; epigenetics; argininosuccinate synthetase; argininosuccinate lyase; autophagy

Abbreviations: ASL, argininosuccinate lyase; ASS, argininosuccinate synthetase; ADI-PEG20, arginine deiminase PEG20; 5'AZA, 5-Aza-2'-deoxycytidine; GBM, glioblastoma multiforme; qPCR, quantitative PCR; CSC, cancer stem cell; MSP, methylation-specific PCR; SRB, sulphorodamine B; CQ, chloroquine

Received 15.6.12; revised 03.11.12; accepted 26.11.12; Edited by P Salomoni
} 
in activated rat microglia, ${ }^{23,24}$ implying that both genes are dynamically controlled according to cellular stresses. So far, no reports of ASL-negative cancers have been documented.

Previous studies have identified a number of genes subject to methylation-dependent transcriptional silencing in highgrade brain tumours. ${ }^{25-27}$ To seek additional epigenetically regulated genes in GBM, we performed methylation reversal and micro-array analysis in GBM cell lines. Here, we describe the identification and characterization of the two key enzymes in arginine biosynthesis, ASS1 and ASL, as epigenetically regulated genes in GBM, and we demonstrate that epigenetic changes in these changes modulate cellular sensitivity to therapeutic autophagy.

\section{Results}

Methylation reversal identifies novel methylated genes in GBM. We used 5-Aza-2'-deoxycytidine (5'AZA) to reverse transcriptional silencing in the GAMG GBM cell line. mRNAs upregulated by demethylation were identified by micro-array analysis. The mRNA of 47 genes was upregulated at least twofold by $5^{\prime} \mathrm{AZA}$ and of 791 genes at least 1.4-fold. We selected 10 genes and confirmed upregulation by $5^{\prime} A Z A$ for 7 of them (Figure 1a). Among the genes upregulated by $5^{\prime} \mathrm{AZA}$ in GAMG cells was ASS1, which contains a $C p G$ island in the $5^{\prime}$ regulatory sequences of the gene (see Supplementary Figure S1 for map of CpG island, including location of primers). This gene encodes ASS, the enzyme catalysing the rate-limiting step in arginine biosynthesis. Using quantitative PCR (qPCR) and western blotting, we confirmed upregulation of ASS1 mRNA by $5^{\prime} A Z A$ in the GAMG cell line but not in the 42MG cell line (Figure 1b). Using methylation-specific PCR (MSP), we confirmed that the increased expression of ASS1 in GAMG following $5^{\prime} \mathrm{AZA}$ is accompanied by a decrease in $\mathrm{CpG}$ island methylation that does not occur in 42MG (Figure 1b).

Silencing of ASS1 in primary cultures of GBM. To investigate in detail the potential epigenetic regulation of ASS1, we established 22 primary GBM cultures from tissue obtained at first surgical debulking of patients in our clinical practice. These early passage cultures were tested for expression and methylation of ASS1. Downregulation of ASS1 mRNA and protein was observed in 8/22 cases, results are shown for 10 cases (Figure 1c). Using MSP and pyrosequencing, all cases with ASS1 methylation had downregulation of ASS1 mRNA (GBM 31, 53 and 59). However, in some cases, ASS1 mRNA was downregulated
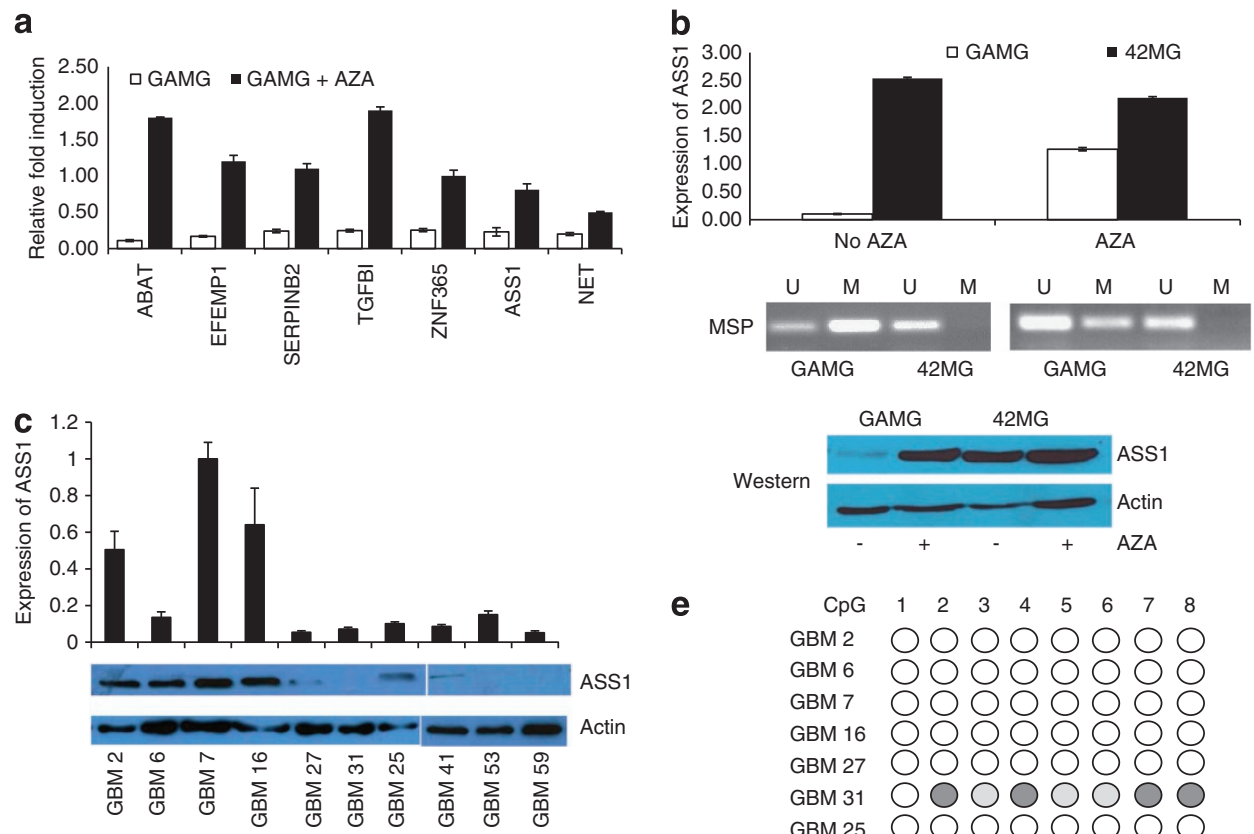

e
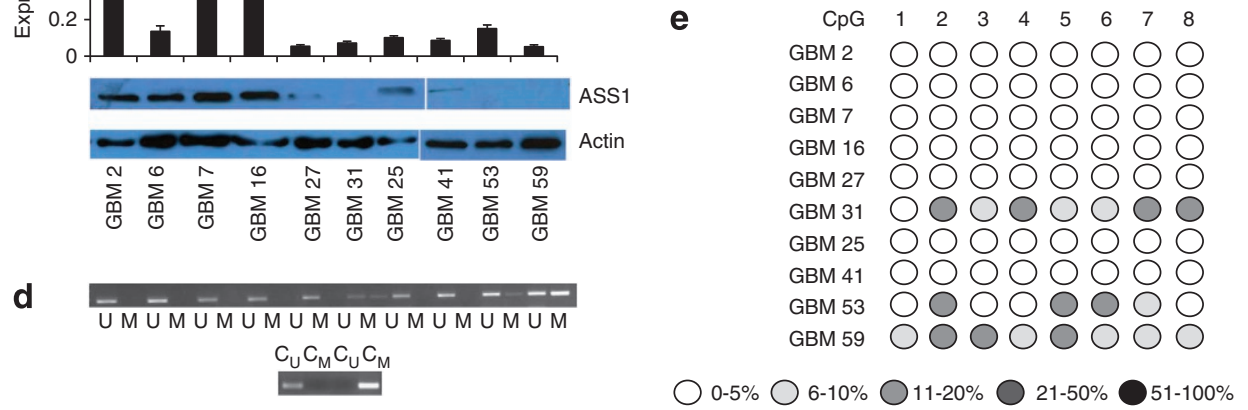

Figure 1 Methylation-dependent transcriptional silencing of ASS1 in GBM. (a) Expression of novel candidate genes is upregulated by demethylation. The figure shows qPCR analysis of the indicated genes in GAMG cells treated (black) or untreated (clear) with $5^{\prime} A Z A$. Experiments were performed in triplicate and data shown are mean fold increases (+ I - 1 S.D.) in 5'AZA-treated cells relative to control cells treated with dimethylsulphoxide. (b) $5^{\prime}$ AZA upregulates ASS1 in GAMG but not $42 M G$ cells. The top panel shows qPCR, the middle panel MSP and the bottom panel western blot analysis of ASS in GAMG and 42MG cells with and without exposure to $5^{\prime}$ AZA as indicated. Actin is used as a loading control for the western blot. (c) qPCR and western blot analysis of ASS1 in primary GBM explants. qPCR was performed in triplicate and data shown are expression relative to GBM 7 ( + / - 1S.D.). (d) MSP analysis of ASS1 CpG island in primary GBM explants. The figure shows unmethylated (U) and methylated (M) reactions for each case. Also shown are control $U$ and M DNA samples modified in parallel with the experimental DNA samples. (e) Pyrosequencing analysis of ASS1 CpG island in primary GBM explants. The level of methylation in individual $\mathrm{CpG}$ dinucleotides is indicated by the intensity of shading as shown 
but without detectable methylation in the CpG island (GBM 6 27, 25 and 41; Figures 1d and e).

ASL is silenced in primary GBM. ASS catalyses the rate-limiting step in arginine biosynthesis prompting us to ask whether expression of ASL, the next enzyme in the arginine biosynthetic pathway, is also downregulated in GBM. As no antibodies recognizing $A S L$ protein exist, we analysed expression of $A S L$ using qPCR. Downregulation of $A S L$ mRNA was observed in 5/22 primary GBM cultures, results are shown for 10 primary cultures (Figure 2a). As with ASS1, there is a $\mathrm{CpG}$ island in the regulatory sequences of $A S L$ (Supplementary Figure S1). Using MSP and pyrosequencing, we showed that each of the primary GBM with downregulation of $A S L$ was methylated in the $A S L C p G$ island (Figures $2 \mathrm{~b}$ and $\mathrm{c}$ ). To confirm the role of $\mathrm{CpG}$ island methylation, we treated cells with $A Z A$ and observed upregulation of $A S L$ in GBM 59 (CpG island methylated) but no effect on $A S L$ levels in GBM 6 (CpG island unmethylated). Following $A Z A$, there was a reduction in CpG island methylation in GBM 59 (Figure 2d).

As was observed for ASS1, there were cases in which ASL mRNA was downregulated but without detectable methylation in the CpG island (GBM 16 and 41).

\section{Methylation abrogates adaptive transcriptional upregulation of $A S S 1$ and $A S L$ and confers arginine auxotrophy. As ASS1 and ASL are key enzymes in the biosynthesis of arginine, we tested the effects of arginine deprivation on the growth of primary GBM cultures using the}

enzyme ADI-PEG20. We first performed a detailed dose response analysis and showed that the presence of $\mathrm{CpG}$ island methylation in either ASS1 or ASL CpG island was associated with sensitivity to the anti-proliferative effects of ADI-PEG20 (GBM 31, 27), whereas cells in which the CpG islands of $A S S 1$ and $A S L$ were unmethylated were insensitive to ADI-PEG20 (GBM 16) (Figure 3c and Supplementary Table S2). Cells with methylation in both $\mathrm{CpG}$ islands were hypersensitive to the drug, with complete inhibition of growth at a concentration of $0.06 \mu \mathrm{g} / \mathrm{ml}$ (GBM 59) (Figure 3c). For analysis of $A S S 1$ and $A S L$ gene expression, qPCR and western blotting were performed $48 \mathrm{~h}$ post treatment. ADIPEG20 induced robust upregulation of $A S S 1$ and $A S L$ mRNA and ASS protein in unmethylated lines as shown in Figures $3 \mathrm{a}$ and $\mathrm{b}$, respectively. This adaptive upregulation was absent in cells with $\mathrm{CpG}$ island methylation, but upregulation was readily induced in these cells by $5^{\prime} A Z A$. These results suggest that $\mathrm{CpG}$ island methylation in $A S S$ and $A S L$, rather than absolute expression levels per se, is the critical determinant of sensitivity to ADI-PEG20.

To confirm these results, a panel of GBM cell lines were similarly tested. We confirmed that both ASS1 and ASL are subject to methylation-dependent transcriptional silencing (Supplementary Figure S2) and that CpG island methylation is a critical determinant of sensitivity to ADI-PEG20 (Supplementary Figure S3 and Supplementary Table S2).

Knock down confirms the role of ASS1 in ADI-PEG20 sensitivity. To further verify the role of $A S S 1$ in sensitivity to ADI-PEG20, we generated stable knockdown cells of ASS1

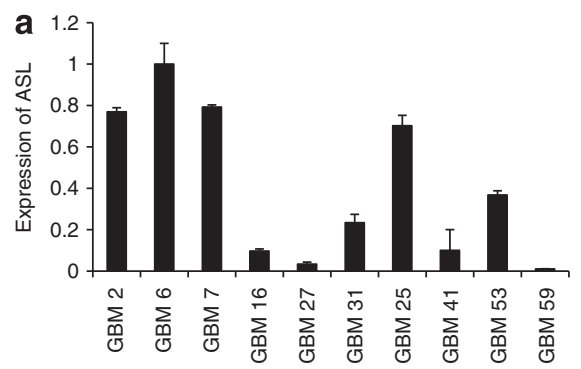

C
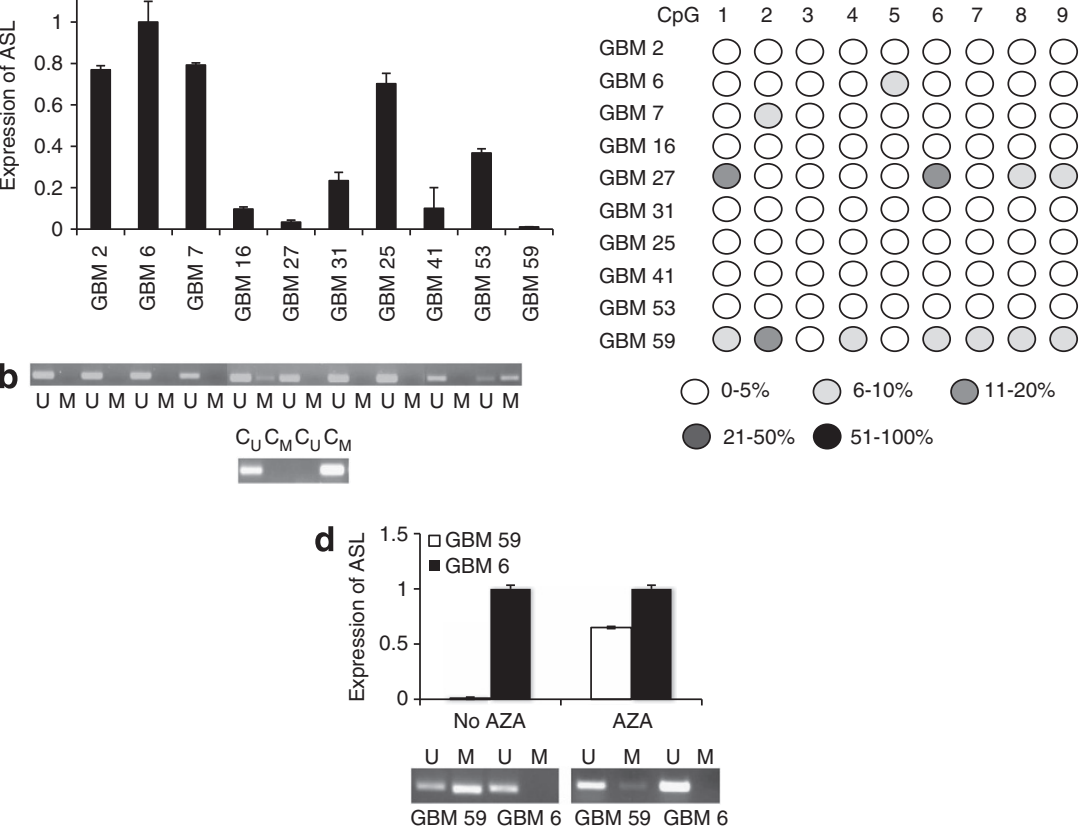

Figure 2 Methylation-dependent transcriptional silencing of ASL. (a) qPCR analysis of ASL in primary GBM explants. qPCR was performed in triplicate and data shown are expression relative to GBM $6(+/-1 \mathrm{SD})$. (b) MSP analysis of $A S L \mathrm{CpG}$ island in primary GBM explants. The figure shows unmethylated (U) and methylated (M) reactions for each case. Also shown are control $U$ and M DNA samples modified in parallel with the experimental DNA samples. (c) Pyrosequencing analysis of $A S L$ CpG island in primary GBM explants. The level of methylation in individual CpG dinucleotides is indicated by the intensity of shading as shown. (d) $5^{\prime} \mathrm{AZA}$ upregulates ASL in primary GBM 59 but not in primary GBM 6 cells. The top panel shows qPCR and the bottom panel MSP analysis of ASL in GBM 59 and GBM 6 cells with and without exposure to $5^{\prime} A Z A$ as indicated 

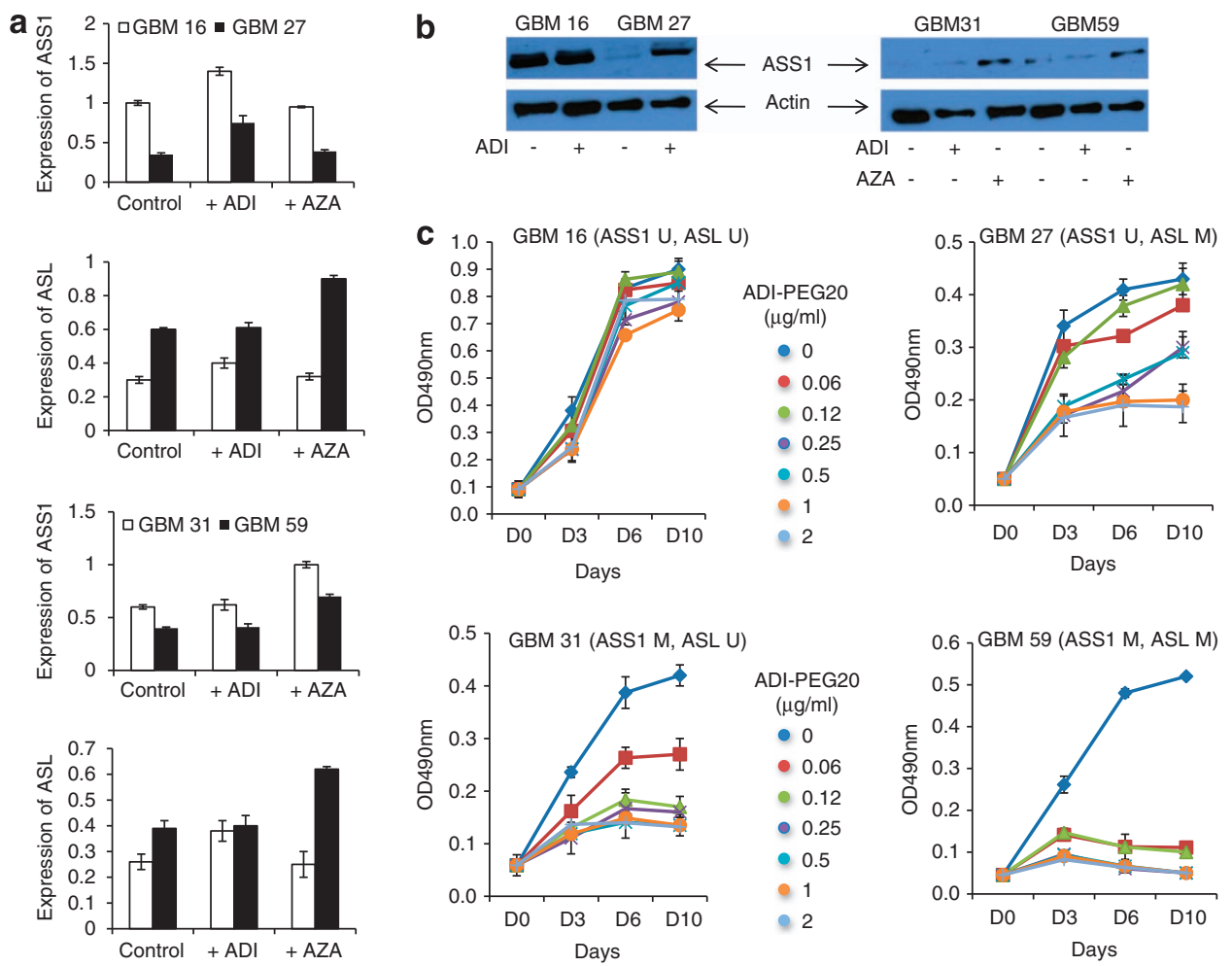

Figure 3 Methylation in the ASS1 and ASL CpG islands blocks transcriptional upregulation upon arginine deprivation and confers arginine auxotrophy and sensitivity to arginine deiminase (ADI-PEG20) in primary GBM explants. (a) Arginine deprivation induces ASS1 and ASL mRNA in primary GBM cells, but this is abrogated by CpG island methylation. The indicated GBM explants were grown in the presence of ADI-PEG20 $(1 \mu \mathrm{g} / \mathrm{ml})$ or $5^{\prime} \mathrm{AZA}(1 \mu \mathrm{M})$ as shown. RNA was harvested after $48 \mathrm{~h}$ and subjected to qPCR analysis of $A S S 1$ and $A S L$ as indicated. Each experiment was performed at least twice and the values shown are means $(+/-1$ SD) relative to untreated control cells. (b) Western blot analysis of ASS in primary GBM cells. The indicated primary explants were grown in the presence of ADI-PEG20 $(1 \mu \mathrm{g} / \mathrm{ml})$ or $5^{\prime} \mathrm{AZA}(1 \mu \mathrm{M})$ as shown. ASS levels were analysed by western blotting after $48 \mathrm{~h}$. Actin is used as a control protein. (c) Dose response curves for ADI-PEG20 in primary GBM of varying CpG island methylation status. Logarithmic phase primary GBM cells were exposed to the indicated concentrations of ADI-PEG20 and proliferation assessed by measurement of SRB as described in Methods. The most sensitive tumour is GBM 59 in which both ASS1 and ASL CpG islands are methylated and which is fully inhibited by $0.06 \mu \mathrm{g} / \mathrm{ml}$ ADI-PEG20. By contrast, GBM16 (ASS1 and ASL unmethylated) is unaffected by ADI-PEG20. GBM 27 and GBM 31 (either ASS1 or ASL CpG islands methylated) show intermediate sensitivity to ADI-PEG20

using the T98G cell line. Having confirmed knock down by qPCR and western blotting (Figure $4 a$, upper panel), we showed that reduced levels of ASS1 confer sensitivity to ADI-PEG20 (Figure 4a, lower panel).

Arginine auxotrophic GBM CSCs are sensitive to ADI-PEG20. The presence of CSCs in GBM is thought to be associated with resistance to temozolomide and treatment failure. ${ }^{6,28}$ Targeting these cells is therefore critical. Using flow cytometry, we detected a population of stem cells in the DBTRG GBM cell line (ASL methylated, partial sensitivity to ADI-PEG20) using the putative stem cell marker, CD133 (Figure 4b, upper panel). To test the effect of ADI-PEG20 on the CD133 ${ }^{+}$component, sorted cells were challenged with ADI-PEG20 and analysed for proliferation. Both populations of cells were equally inhibited in the presence of ADI-PEG20 (Figure 4b, lower panel).

Methylation of ASS1 sensitizes cells to autophagy upon arginine deprivation. Previous studies have shown that ADI-PEG20 induces autophagy in some ASS-negative cell lines, ${ }^{22,29}$ but the relationship to epigenetic status is unknown. As autophagy is a protective response to conditions of nutrient deprivation, we were interested to determine whether ADI-PEG20 was initially inducing an autophagic response in GBM cells. To investigate this, cell lines were exposed to ADI-PEG20 and analysed for markers of autophagy by western blotting (upregulation of Beclin 1 and the Atg genes 5 and 7 , degradation of $\mathrm{P} 62$ and the conversion of cytosolic LC3-1 to the lipidated form LC3$11^{30}$ ) and acridine orange staining (formation of acidic vesicular organelles). Acidic vesicular organelles are a characteristic feature of autophagy and can be detected and measured by vital staining with acridine orange, which accumulates in these acidic compartments, and is detectable as bright red fluoresence. ${ }^{31}$ Typical autophagic changes were detectable as early as $6 \mathrm{~h}$ after ADI-PEG20 treatment and progressively accumulated at 16 and $24 \mathrm{~h}$ in the ASS1methylated line LN229 only (Figure 4c). Similarly, acidic vesicular organelles were only detected in this line and not in the unmethylated line T98G (Figure 4d). Treatment with bafilomycin $A 1$, a specific inhibitor of vacuolar-type $\mathrm{H}^{+}$ ATPase, abrogated the formation of acidic vesicular organelles and no fluorescence was observed (Figure 4d). 
a
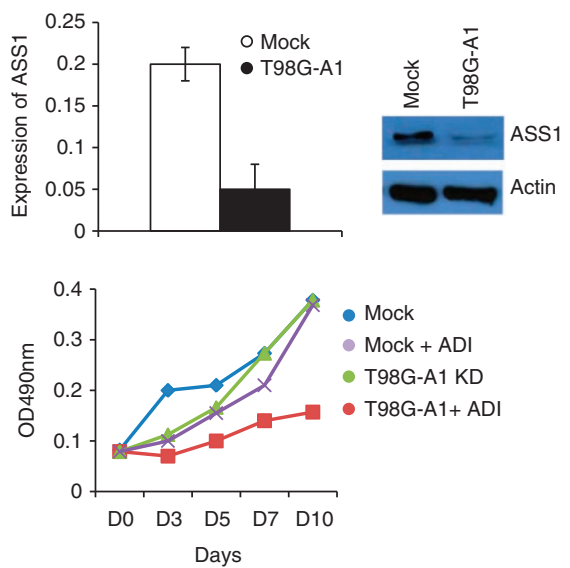

b

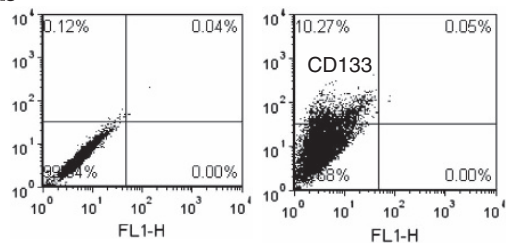

- CD133 +ve - CD133 +ve + ADI

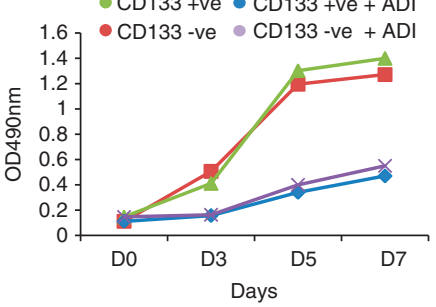

c
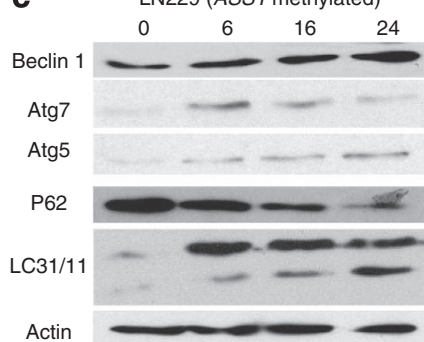

T98G (ASS1 unmethylated)

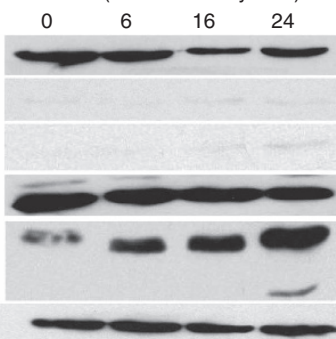

d

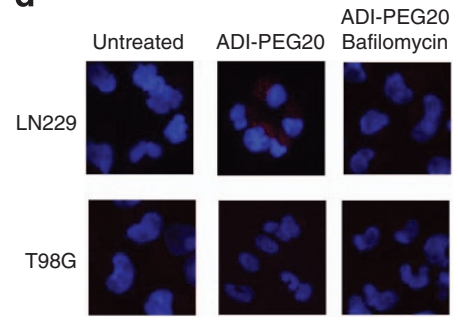

Figure 4 ADI-PEG20 kills ASS1 knockdown cells, ASL negative CSCs and induces autophagy. (a) ASS1 knockdown cells are sensitive to ADI-PEG20. Stable ASS1 knockdown cells (T98G-A1) were exposed to ADI-PEG20 and analysed for proliferation as described in Methods. Cells containing empty vector were treated similarly. Upper panel confirms knock down of ASS1 by qPCR and western blotting. T98G-A1 cells are more sensitive to killing than mock transfected cells, lower panel. (b) ADI-PEG20 kills CD133-positive GBM cells. DBTRG cells (ASS1 unmethylated, ASL methylated) were sorted for CD133-positive cells (left histogram, lgG isotype control PE antibody; right histogram, anti-CD133 showing $10.27 \%$ CD133-positive cells). CD133-positive and -negative fractions were then exposed to ADI-PEG20 (1 $\mu \mathrm{g} / \mathrm{ml})$ and proliferation assessed by SRB staining. CD133-positive and -negative fractions are equally sensitive to inhibition by ADI-PEG20, lower graph. (c and d) ADI-PEG20 induces autophagic markers. LN229 and T98G cells were exposed to ADI-PEG20 $(1 \mu \mathrm{g} / \mathrm{ml}$ ) and analysed for autophagic markers by western blotting (upregulation of Beclin 1 and Atg proteins, degradation of P62 and conversion of LC3-1 to LC3-11) (c) and by acridine orange staining (formation of acidic vacuoles) in the presence or absence of bafilomycin $48 \mathrm{~h}$ after treatment (d). Autophagic changes are observed in the ASS1 methylated cell line, LN229, only. Bafilomycin abrogates the formation of acidic vacuoles, which stain red in the absence of bafilomycin

Chloroquine (CQ) inhibits autophagy and accelerates ADI-PEG20 induced cell death. To test the hypothesis that ADI-PEG20 induced autophagy is (at least initially) a survival strategy for GBM cells, we investigated the effect of $C Q$, an inhibitor of autophagy. CQ efficiently blocked ADI-PEG20induced p62 degradation and increased LC3-1 to LC3-11 conversion in the ASS1-methylated cell line LN229 (Figure $5 \mathrm{a}$ ). These results are consistent with the fact that $\mathrm{CQ}$ acts late in autophagy, resulting in the accumulation of lipidated LC3-11. To further verify autophagy, stable LN229 cell lines expressing GFP-LC3 were generated to visualize autophagosome formation in real time in live cells. During autophagasome formation, GFP-LC3B is processed and recruited to the autophagosome membrane where it can be imaged as cytoplasmic puncta by high resolution fluorescence microscopy. Punctate GFP staining could be detected in ADI-PEG20-treated cells, which accumulated in the presence of $C Q$. This is consistent with the fact that $C Q$ inhibits autophagic flux of LC3 (Figure 5b). No punctate staining was observed in GFP vector control cells.

To examine the effect of CQ on ADI-PEG20-induced cell death, cells were treated with either or both $C Q$ and
ADI-PEG20 and analysed by flow cytometry for sub-G1 content using propidium iodide. CQ accelerated and enhanced ADI-PEG20-induced cell death in cells with methylation in either the ASS1 or ASL CpG island as evidenced by an increase in the sub-G1 compartment (Figure 5c). By contrast, there was no effect of $C Q$ in cells lacking methylation in the $A S S 1$ and $A S L$ CpG islands (Figure $5 \mathrm{c}$ ). In methylated lines, cell death was first detectable at $72 \mathrm{~h}$ following treatment with ADI-PEG20 alone. However, in combination with $C Q$, cell death could be detected at $48 \mathrm{~h}$. By $72 \mathrm{~h}, \mathrm{CQ}$ further increased ADI-PEG20-induced cell death (Figure 5c).

Caspase inhibitors do not prevent ADI-PEG20-induced cell death. To determine the role of caspases in ADIPEG20-induced cell death, a caspase inhibition assay was performed. LN229 (ASS1 methylated) and T98G (ASS1 and $A S L$ unmethylated) cells were pre-treated with the pan caspase inhibitor Z-VAD and then exposed to ADI-PEG20. Cells were then harvested for assessment of caspase- and non-caspase-mediated cell death by staining with FITCannexin and propidium iodide. Treatment of cells with Z-VAD 

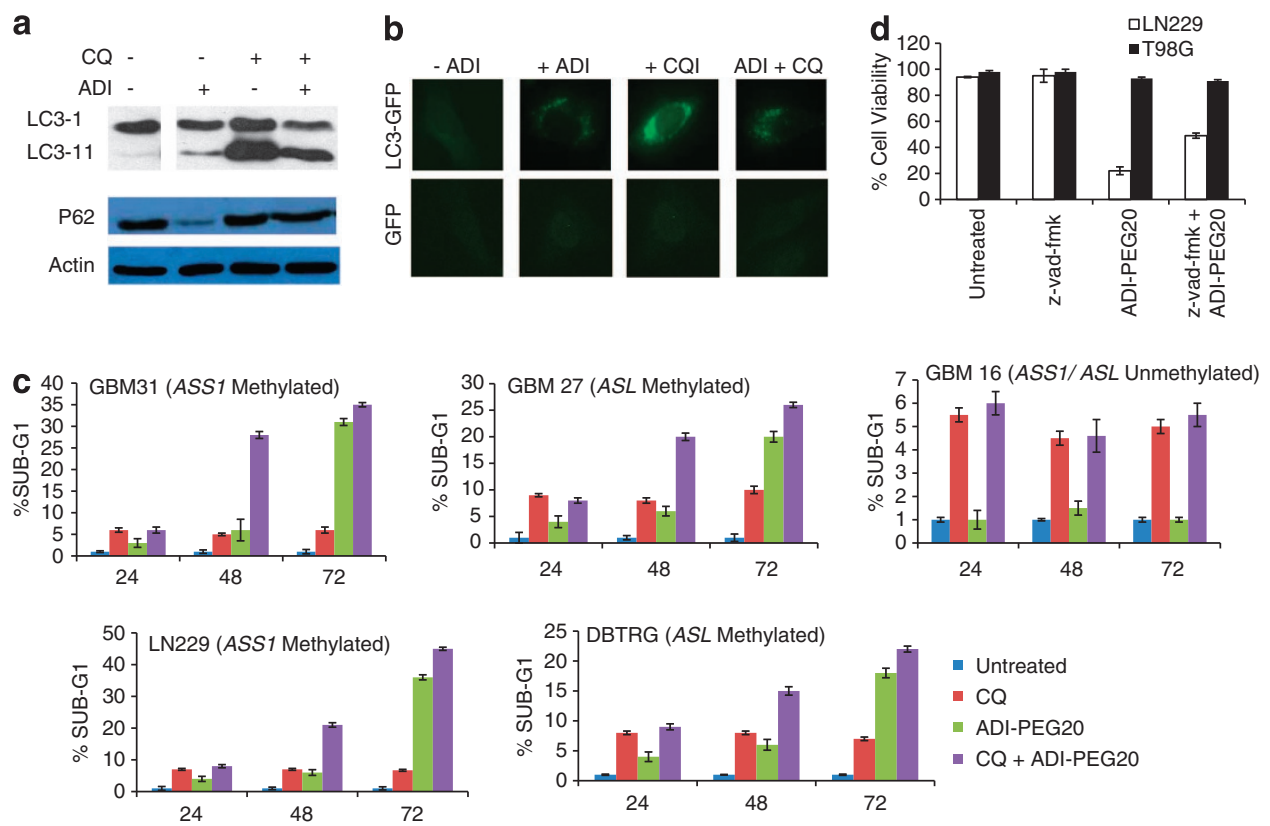

Figure 5 Caspase-independent cell death is induced by ADI-PEG20 and is accelerated by chloroquine. (a) CQ inhibits autophagic markers. LN229 cells were treated with $1 \mu \mathrm{g} / \mathrm{ml} \mathrm{ADI-PEG20} \mathrm{with} \mathrm{and} \mathrm{without} 10 \mu \mathrm{g} / \mathrm{ml} \mathrm{CQ}$ and analysed for conversion of LC3-1 to LC3-11 and degradation of P62 by western blotting $48 \mathrm{~h} \mathrm{post} \mathrm{treatment.} \mathrm{CQ} \mathrm{inhibits}$ degradation of P62 and prevents autophagic flux as evidenced by accumulation of the LC3-11 band. (b) LN229 cells stably expressing GFP-LC3 or empty GFP vector were treated as above and analysed for punctate, autophagosome-associated LC3-11 GFP by fluorescence microscopy. (c) Inhibition of autophagy accelerates and enhances ADIPEG20 induced cell death. Primary and GBM cell lines were treated with $10 \mu \mathrm{M} \mathrm{CQ}, 1 \mu \mathrm{g} / \mathrm{ml}$ ADI-PEG20 or in combination and analysed for sub G1 content by flow cytometry at various time points. (Primary lines - GBM 31 ASS negative, GBM 27 ASL negative, GBM 16 ASS and ASL positive; GBM cell lines - LN229 ASS negative, DBTRG ASL negative). CQ in combination with ADI-PEG20 accelerates and enhances cell death in ASS1/ASL negative lines only as evidenced by an increase in the sub-G1 compartment. The results shown are mean values from three independent experiments. (d) Caspase inhibitors do not prevent ADI-PEG20-induced cell death. LN229 and T98G cells were exposed to $1 \mu \mathrm{g} / \mathrm{ml}$ ADI-PEG20 and analysed for sub-G1 content as above. Where indicated, the caspase inhibitor Z-VAD-fmk was added to cells $24 \mathrm{~h}$ before addition of ADIPEG20. Results are expressed as the mean percentage of live cells remaining from three independent experiments. ADI-PEG20 has no effect on cell death in the unmethylated cell line, T98G but induces marked cell death $(78 \%)$ in the methylated line, LN229. The percentage of cell death induced by ADI-PEG20 is only partially blocked by Z-VAD-fmk $(51 \%)$

alone had no effect on cell death in either LN229 or T98G (Figure 5d). Caspase-independent cell death was observed in LN229 $(51 \%)$ but not T98G cells when pre-treated with Z-VAD in the presence of ADI-PEG20. There was a higher percentage of cell death in LN229 treated with ADI-PEG20 alone $(78 \%)$, suggesting that $27 \%$ of cell death is attributable to caspases.

ASS1 and $A S L$ are frequent targets for epigenetic inactivation in clinical cases of GBM. In order to translate these results into the clinical setting, we examined the two genes in clinical cases of human GBM using immunohistochemistry, MSP and pyrosequencing. Previous studies on ASS in melanoma, ovarian carcinoma and osteocarcinoma showed focal expression in tumour tissue. There are no published data on the expression of ASL in tumour tissue. Sixty patients (36 male, 24 female, median age 57 years) were retrospectively studied for $A S S$ and $A S L$ methylation and, of these cases, 55 were evaluated for ASS expression and 57 for ASL expression using a semi-quantitative scoring system as described in Methods. Representative ASS and ASL immunostaining and MSP in clinical cases are shown in Figures $6 \mathrm{~b}$ and $\mathrm{d}$. The methylation status of primary cells generated from some of these primary tumours is also shown (Figure 6d). These primary lines were stained for GFAP, a putative astrocyte marker and H\&E stained (Figure 6a). All cases were further characterized by staining for $\mathrm{p53}$, IDH1 and EGFR, CD133 and MGMT methylation (data not shown).

In all, 13/55 samples were negative for ASS expression by immunohistochemistry and scored 0 . These negative cases correlated well with MSP and pyrosequencing data. A further 15 cases were methylated by MSP and had pyrosequencing values $>5$, an arbitrary cutoff for methylation positivity. Eleven of these cases had low ASS staining (score 1), whereas four cases exhibited strong ASS staining (score 2). These results are represented in Supplementary Table S3. Similarly for ASL, $5 / 57$ stained negative (score 0 ) and correlated with methylation. A further five showed no correlation with methylation and immunohistochemistry. Eight cases were positive for both ASS1 and ASL methylation (Supplementary Table S3).

ASS1 and ASL CpG island methylation is a predictive biomarker in GBM. To determine whether methylation of ASS1 and/or ASL affects outcomes of patients, we carried out a statistical analysis as described in Methods. Median overall survival for GBM patients with unmethylated ASS1 CpG island was 496 days versus 309 days for patients with methylated ASS1 $\mathrm{CpG}$ island $(P=0.11)$. Median overall survival for patients with unmethylated $A S L \mathrm{CpG}$ island was 

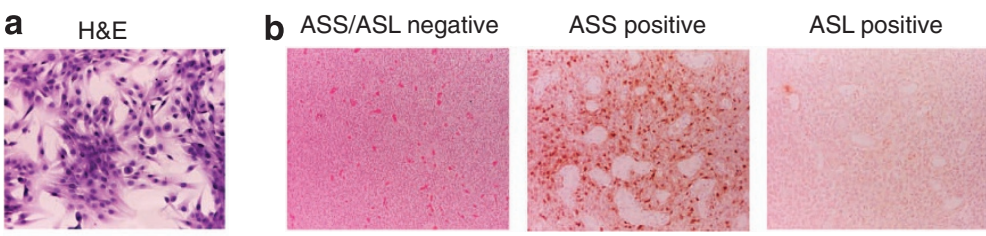

GFAP
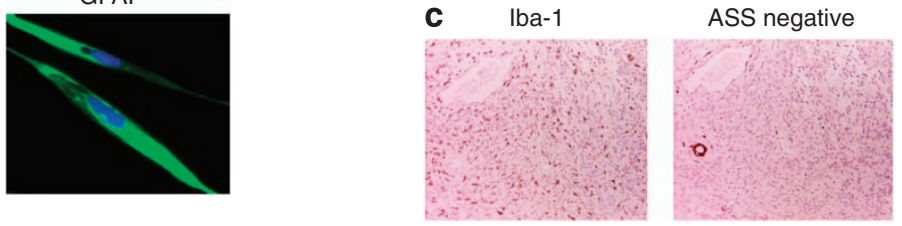

d
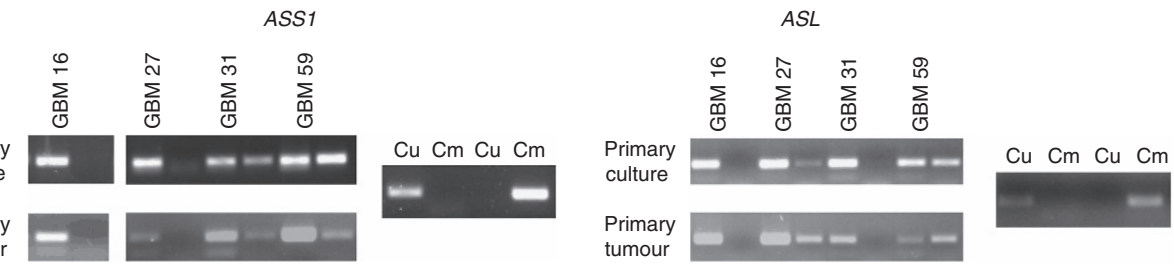

Figure 6 ASS and ASL in clinical cases of GBM. (a) Primary lines generated from tumour explants were stained for GFAP and hematoxylin and eosin. Representative examples are shown. (b) Immunohistochemistry showing ASS and ASL in clinical cases of GBM. (c) Immunohistochemical analysis for the microglial marker, Iba-1, in ASSnegative GBM. (d) MSP for ASS1 and ASL CpG islands in clinical cases of GBM and primary lines generated from them. The figure shows unmethylated (U) and methylated (M) reactions for each case. Also shown are control $U$ and M DNA samples modified in parallel with the experimental DNA samples

443 days compared with 299 days for patients with methylated $A S L$ CpG island $(P=0.16)$. Median overall survival for patients whose GBM showed methylation in the CpG islands of both ASS1 and ASL was significantly shorter than those in whom neither CpG island was methylated or only one of the two CpG islands was methylated. Median overall survival in cases with either $\mathrm{CpG}$ island or none methylated $=436$ days versus 299 days $(P=0.0468)$ (Figure 7).

ASS/ASL in non-neoplastic cells. Gliomas are characterized by a high number of non-neoplastic microglial cells residing within and around the tumour. Previous studies on rat microglia showed that ASS and ASL proteins are upregulated in response to damage. ${ }^{24}$ We were interested to determine the ASS1/ASL status of the infiltrating microglia as they could effectively provide an exogenous source of arginine for ASS/ASL-negative tumours and negate the effect of ADI-PEG20 treatment. Tissue sections were stained for Iba-1, a macrophage/microglia-specific surface antigen and for ASS. No ASS staining was observed in lba-1-positive cells, suggesting that microglia within GBM do not/cannot upregulate the ASS1 gene (Figure 6c).

\section{Discussion}

Despite advances in imaging and surgical techniques and the use of temozolomide, GBM remains a universally and rapidly fatal cancer, affecting patients of all ages. As such, the requirement for novel therapeutic strategies is clear and pressing. Here we show that $A S S 1$ and $A S L$, encoding the two key enzymatic components of the arginine biosynthetic pathway, are epigenetically regulated in glial brain tumours, and we provide evidence that targeting of this pathway is a novel therapeutic strategy for a subset of patients with this disease. The importance of metabolic changes in brain tumours has been recently illustrated by the detection of gain-of-function mutations in isocitrate dehydrogenase in oligodendrogliomas and the secondary GBM, which develop from a subset of them. ${ }^{32}$ The present data show that arginine metabolism is disrupted in a proportion of GBM, further emphasizing that abnormalities in fundamental metabolic pathways are a major feature of this disease. A key element of our study is the use of low passage primary cultures of GBM cells. These cultures are established in culture on the day of surgical debulking, closely resemble the primary GBM from which they were established in both expression and methylation of $A S S 1$ and $A S L$ and in their response to arginine deprivation, analogous to established GBM cell lines and are clearly a more appropriate model for pharmacological studies than cell lines that have been maintained in culture for many years.

We initially identified $A S S 1$ as a transcriptionally downregulated gene in studies using the demethylating agent $5^{\prime} \mathrm{AZA}$ to reverse methylation. A number of genes were upregulated that have not been previously described as epigenetically regulated in brain tumours specifically or, more generally, in human cancer. These include, among others, amino butyrate amino transferase $(A B A T), E F E M P 1$ and ZNF365. The significance of silencing of these genes and their role (if any) in the pathogenesis of GBM awaits further analysis.

We show, using two independent techniques (MSP and pyrosequencing), that the ASS1 CpG island is aberrantly methylated in $30 \%$ and the ASL CpG island in $22 \%$ of GBM. Only a single study has previously demonstrated 

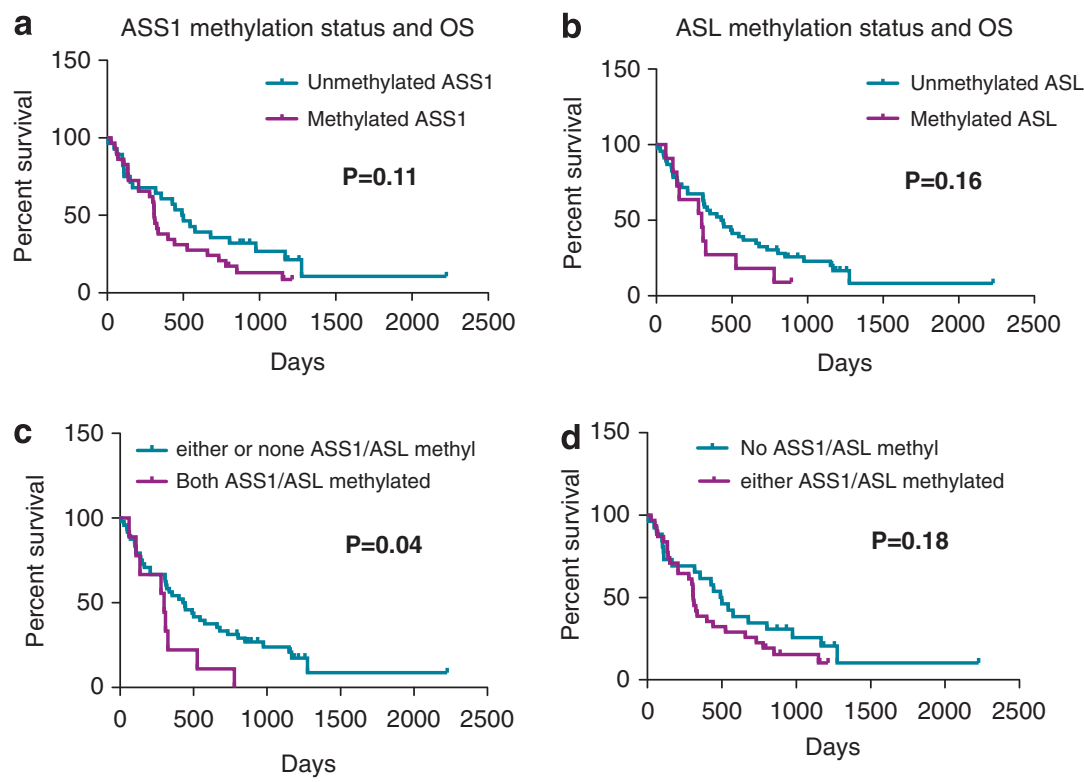

Figure 7 Methylation of ASS1 and ASL CpG island is a predictive biomarker in GBM. To determine whether methylation of $A S S 1$ and/or ASL affects outcomes of patients, Kaplan-Meier curves were used to estimate the probabilities of survival and the log-rank test to assess the statistical significance of differences in event rates using Prism 5. (a) Median overall survival (OS) for GBM patients with unmethylated ASS1 CpG island was 496 days versus 309 days for patients with methylated ASS1 CpG island $(P=0.11)$. (b) Median OS for patients with unmethylated $A S L C p G$ island was 443 days compared with 299 days for patients with methylated $A S L C p G$ island $(P=0.16)$. (c) Median OS for patients whose GBM showed methylation in the CpG islands of both ASS1 and ASL was significantly shorter than those in whom neither CpG island was methylated or only one of the two CpG islands was methylated $(P=0.0468)$. (d) Median OS in cases with either $\mathrm{CpG}$ island or none methylated $=436$ days versus 299 days $(P=0.18)$

methylation-dependent transcriptional silencing of ASS1 in neoplasia, this in platinum-resistant epithelial ovarian cancer, ${ }^{21}$ but no previous study has examined expression and epigenetic regulation of $A S L$. As such, ours is, to the best of our knowledge, the first demonstration of epigenetic regulation of this gene in human neoplasia. Silencing of ASS1 and $A S L$ implies strong selective pressure to downregulate the arginine biosynthetic pathway in GBM. Moreover, we have shown that simultaneous methylation in the $\mathrm{CpG}$ islands of both ASS1 and ASL occurs in 14\% of GBM cases in our series, further emphasizing that strong selective pressure against cancer cells expressing the two genes must operate in GBM.

In GBM cells lacking $\mathrm{CpG}$ island methylation, the mRNA of both $A S S 1$ and $A S L$ is upregulated by exposure to ADI-PEG20 and a similar upregulation is seen when cells are grown in arginine-free culture medium (data not shown). Upregulation of biosynthetic enzymes has been long recognized as a normal physiological response to nutritional deprivation, for example, under conditions of starvation. ${ }^{28,29}$ Here we show that $\mathrm{CpG}$ island methylation blocks the adaptive transcriptional response to arginine deprivation and it is this, rather than absolute expression levels of ASS or ASL, that is the critical determinant of cellular sensitivity to ADI-PEG20.

We demonstrate herein that ADI-PEG20 kills GBM cells with $A S S / A S L$ CpG island methylation predominantly independently of caspase activation, suggesting that failure of apoptosis will not be a barrier to successful therapy with ADIPEG20. Furthermore, inhibiting autophagy by CQ markedly accelerated cell death by ADI-PEG20, implying that autophagy triggered by ADI-PEG20 is initially a protective response.
Trials of the clinical formulation of ADI-PEG20 are in progress in advanced hepatocellular carcinoma, relapsed or refractory small cell lung cancer and malignant pleural mesethelioma (http://clinicaltrials.gov). A number of factors from our work suggest that clinical trial of ADI-PEG20 in GBM is merited. First, a major advantage of the proposed study in GBM is that patients whose cancers exhibit methylation of ASS1 and/or ASL can be further pre-tested for sensitivity to ADI-PE20 in vitro using primary cultures to inform the physician of likely treatment response. Such primary cultures can be established efficiently, rapidly and with a high probability of success from tissue obtained at biopsy or primary debulking. Importantly, sufficient cells to allow predictive pharmacological testing are available within a credible time scale after surgery to inform the use of ADIPEG20. Second, the extremely unfavourable prognosis for GBM patients correlates with intrinsic resistance to apoptosis of GBM cells. ${ }^{4,5}$ Clinical trials of $\mathrm{CQ}$ in combination with standard therapy showed some improvement in the survival of GBM patients. ${ }^{30,31}$ Interestingly, the $C Q$ derivatives quinacrine and mefloquine show superior blood/brain penetration in comparison to $C Q$ and are more potent in vitro than $C Q$ in killing GBM cells, ${ }^{33}$ and we propose that combinations of ADIPEG20 with $C Q$ or $C Q$ derivatives merit consideration for clinical trial in appropriate GBM patients. Third, recurrence of GBM after therapy has been attributed to the presence of chemo-/radio-resistant CD133 ${ }^{+} \mathrm{CSCs}^{6}$ We have shown in this work that in cells with methylated $A S L, \mathrm{CD}_{133^{+}} \mathrm{GBM}$ cells are growth inhibited by ADI-PEG20 with the same efficiency as $\mathrm{CD}_{133^{-}}$, implying that the presence of chemo-/ radio-resistant $\mathrm{CD} 133^{+}$cells will not be a barrier to effective therapy with ADI-PEG20 in vivo. Fourth, a major factor limiting 
effective management of GBM is the presence of the blood/ brain barrier, which may act to prevent efficient delivery of chemotherapy into the brain. Clearly, as ADI-PEG20 exerts its therapeutic effect by depletion of peripheral blood arginine, passage across the blood/brain barrier is not required for therapeutic efficacy of this agent. Based on these observations, we believe that clinical trial of arginine-deprivation therapy with $C Q$ or $C Q$ derivative compounds is warranted in GBM. CpG island methylation of either ASS1 or $A S L$, but especially of $A S S 1$ and $A S L$ simultaneously, was associated with particularly poor outcomes in patients treated with the current gold-standard therapy of radiotherapy and temozolomide. We have shown herein that GBM with $\mathrm{CpG}$ island of methylation of both ASS1 and $A S L$ are hypersensitive to ADIPEG20, implying that arginine depletion may be an appropriate therapy for patients with particularly poor prognosis disease.

In conclusion, the data we present here identify a new metabolic defect in GBM cells and suggest a novel therapeutic strategy, which circumvents many of the traditional barriers to effective management of this universally fatal malignancy.

\section{Methods}

Cell lines, primary cultures and clinical cases. Primary GBM cultures were established from fresh tumours and maintained in DMEM/ F12 (1:1) supplemented with $10 \%$ (foetal bovine serum). In brief, fresh tumour material was washed in RPMI/F12 (1:1) and minced through a cell strainer to obtain a singlecell suspension. Contaminating red blood cells were disrupted using sterile $\mathrm{dH} 2 \mathrm{O}$ before cells were transferred to fresh growth medium. Primary cells were expanded and early passage numbers were frozen in liquid $\mathrm{N}_{2}$ for analysis at a later time point. Glioblastoma cell lines were obtained from the German tissue bank (DSMZ) unless otherwise specified and maintained in RPMI (DBTRG), DMEM (GAMG, SNB19, U87, U118, CCF, LN229), MEM (8MG, T87G), DMEM F12 (1:1) (MO59J, MO59K) or MEM/RPMI 1:1 (42MG) supplemented with $10 \%$ foetal bovine serum (All purchased from Invitrogen, Paisley, UK) and $2 \mathrm{mmol} / \mathrm{l}$ L-glutamine (Sigma-Aldrich, Dorset, UK).

Sixty GBM cases selected from the Brain Tumour Registry at Imperial College were selected for detailed analysis of enzymes in the arginine biosynthetic pathway. Detailed clinical history, pre-and post-operative imaging and follow-up were available in 60 cases. In all, 24 patients were female and 36 male with mean age 58 years and 7 months (range $=31$ years and 1 month -83 years 11 months; median 59 years 7 months). In each case, original $\mathrm{H} \& \mathrm{E}$-stained sections were re-examined to confirm the diagnosis, and the most representative samples were used for immunohistochemical stains. Samples were obtained from surgical debulking rather than stereotactic biopsies in order to examine as much tumour tissue as possible. As such, we chose only supratentorial GBMs because gross total debulking is not achievable in tumours occurring in the sub-cortical grey matter, cerebellum, brainstem and spinal cord. Finally, we selected patients with a clinical history consistent with a de novo GBM because almost all patients with secondary GBM received treatment before debulking.

Methylation reversal. Cell lines and primary cultures were grown in the presence of $5 \mu \mathrm{M} 5^{\prime} \mathrm{AZA}$ (Sigma-Aldrich) for 7days. Fresh AZA was added with every medium change and supplemented with $300 \mathrm{nM}$ Trichostatin A (SigmaAldrich) for the last $16 \mathrm{~h}$ of culture. Controls cells were treated with dimethylsulphoxide.

Immunohistochemistry. Paraffin-embedded blocks were cut into $5 \mu \mathrm{m}$ sections and dewaxed in xylene followed by rehydration in a standard alcohol series. Antigen retrieval (for ASS) was achieved by pressure cooking for $20 \mathrm{~min}$ in citrate buffer ( $\mathrm{pH} 6.0$ ), followed by blocking of endogenous peroxidase in $0.3 \%$ $\mathrm{H}_{2} \mathrm{O}_{2}$. Antibodies to ASS (BD Transduction, monoclonal, 25/ASS, 1:500, Oxford, UK), ASL (Abnova, monoclonal, clone 4C5-1F2, 1:200, Heidelberg, Germany) and Iba-1(rabbit polyclonal antibody, Wako, Osaka, Japan, 1:200) were added and incubated overnight at $4{ }^{\circ} \mathrm{C}$ and developed using the Super Sensitive Polymer HRP Detection System (BioGenex Laboratories, Inc., Fremont, CA, USA).
Expression of ASS/ASL was assessed semi-quantitatively where negative tumours are defined as negative with expression ranging between $0-5 \%$ (score 0 ) and diffuse (score 2) those showing expression in $75 \%$ or more tumour cells. Anything in between negative and diffuse was defined focal and scored 1.

Analysis of gene expression in vitro. For $q P C R$, total RNA was prepared from log phase cell lines and primary GBM cultures using the EZNA isolation kit total RNA 1 according to the manufacturer's instructions (VWR, Leicester, UK). cDNA was synthesized from $1 \mu \mathrm{g}$ of total RNA using anchored oligo(dT) primers and MMLV reverse transcriptase (Invitrogen).

Quantitative real-time reverse transcription-PCR (qPCR). All primers were designed using Primer Express version 1.5a software (Life Technologies Ltd, Paisley, UK). qPCR was performed with Syber Green fast mix (Quanta Biosciences, Gaithersburg, MD, USA) using the Biorad CFX96 Detection System instrument and software (Bio-Rad Laboratories Ltd, Hemel Hempstead, UK). In all, $0.5 \mu \mathrm{l}$ cDNA was used per $20 \mu$ l reaction with the following cycling conditions: an initial denaturation at $95^{\circ} \mathrm{C}$ for 3 min was followed by 40 cycles of $95^{\circ} \mathrm{C}$ for $10 \mathrm{~s}, 55^{\circ} \mathrm{C}$ for $30 \mathrm{~s}$ and $72^{\circ} \mathrm{C}$ for $10 \mathrm{~s}$. A melt curve analysis was included as the final step, which consisted of temperature increments of $55-95^{\circ} \mathrm{C}$. Each sample was analysed in triplicate and normalized to GAPDH. Primer sequences for qPCR are given in Supplementary Table S1.

Western blot analysis. Whole-cell extracts were made using RIPA buffer and the protein concentration of lysates estimated using a Bio-Rad Protein Assay kit (Bio-Rad Laboratories Ltd, Hertfordshire, UK). Also, $10 \mu \mathrm{g}$ cell extract was run on a $10 \%$ SDS-acrylamide gel and transferred to a nylon membrane. The membrane was blocked for $2 \mathrm{~h}$ at room temperature (PBS with $0.1 \%$ Tween (PBST) and $5 \%$ milk powder) and probed using appropriate primary antibodies (ASS1, clone 25; BD Biosciences, Oxford, UK, 1:2000 dilution, autophagy antibody sampler kit, Cell Signaling Technology, Danvers, MA, USA) in $0.1 \%$ Tween (PBST) and $5 \%$ milk powder overnight at $4{ }^{\circ} \mathrm{C}$. After washing with $0.1 \%$ Tween (PBST), the membrane was incubated in $0.1 \%$ Tween (PBST) and $5 \%$ milk powder with a horseradish peroxidase-conjugated secondary antibody $(1: 2000$ dilution, room temperature for $1 \mathrm{~h}$ ). The secondary antibody was detected using the ECL Plus Western Blotting Reagent (GE Healthcare, Life Sciences, Buckinghamshire, UK).

Methylation analysis. Methylation of the ASS1 and ASL CpG islands was analysed by MSP and quantitative pyrosequencing. Full maps of the ASS1 and ASL CpG islands are presented in Supplementary Figure S1. Primers for MSP were designed using MethPrimer software. Primer locations are shown in Supplementary Figure S1 and sequences are listed in Supplementary Table S1. Primers were designed to cover the entire region of the $\mathrm{CpG}$ island but only sets 1 , 4, 5 and 7 were used for analysis of methylation (Supplementary Figure S1).

Bisulphite modification and MSP. Bisulphite conversion of genomic DNA was carried out using the Zymo EZ DNA methylation kit (Genetix Ltd, Hampshire, UK). This process converts unmethylated cytosine residues to uracil, whereas methylated cytosine residues remain unchanged. MSP was then carried out to determine the methylation status of ASS1 and ASL. In all, $5 \mu$ l of bisulphitemodified DNA was used as templates for PCR reactions with primers specific for methylated or unmethylated sequences. CpGenome Universal Methylated DNA and CpGenome Universal Unmethylated DNA were used as positive and negative controls, respectively. PCR conditions were as follows: 8 cycles of $95^{\circ} \mathrm{C}$ for $2 \mathrm{~min}$, $51.7^{\circ} \mathrm{C}$ for $30 \mathrm{~s}$ and $72^{\circ} \mathrm{C}$ for $30 \mathrm{~s}$ were followed by 32 cycles of $95^{\circ} \mathrm{C}$ for $30 \mathrm{~s}$, $51.7^{\circ} \mathrm{C}$ for $30 \mathrm{~s}$ and $72^{\circ} \mathrm{C}$ for $30 \mathrm{~s}$, then a final extension at $72^{\circ} \mathrm{C}$ for $5 \mathrm{~min}$. PCR products were electrophoresed through $2 \%$ agarose gels, stained with ethidium bromide and visualized using a transilluminator.

Analysis of CpG methylation by pyrosequencing. Bisulphitemodified genomic DNA (see above) was used as the template in pyrosequencing. Modified DNA was then subjected to bisulphite sequencing. Biotage Sample Prep kit and primers designed to amplify a $145 \mathrm{bp}$ fragment across the $\mathrm{CpG}$ island of ASS1 and ASL genes were optimized for software dedicated to methylation analysis. After pyrosequencing, the analysis was performed using PyromarkID Software (Biotage, Uppsala, Sweden). ASS1 forward 5'-TGTGTTTATAATTTGGG ATGG-3' and reverse $5^{\prime}$-GGGTTTAGAGGAGGAGG-3' primers, ASL forward 5'AGGATTGGAGGATGGAGGTAA-3' and reverse $5^{\prime}$-CCACTAACCAAAACTTTTCT AAC- $3^{\prime}$ primers. Annealing temperatures were $54^{\circ} \mathrm{C}$ for $A S S 1$ and $58^{\circ} \mathrm{C}$ for $A S L$. 
Sensitivity of GBM cells to ADI-PEG20. Cell proliferation was determined at various time points using the sulphorodamine B (SRB) assay (Sigma-Aldrich). ADI-PEG20 was obtained from Polaris Pharmaceuticals Inc. (San Diego, CA, USA) Arginine-free medium was purchased from MP Biomedicals (Cambridge, UK). Cells were seeded in triplicate in 96-well plates at a density of $4 \times 10^{3}$ or $4 \times 10^{4}$ cells per well for cell lines or primary lines, respectively. Twenty-four hours after seeding, the cells were washed 3 times in PBS and cultured in medium containing varying concentrations of ADI-PEG20 $(0-2 \mu \mathrm{g} / \mathrm{ml})$ or in arginine-free medium supplemented with $1 \mathrm{mM}$ citrulline and $2 \%$ dialysed FCS for various time points. Cells were fixed with $10 \%$ tri-chloroacetate for at least $1 \mathrm{~h}$ at $4{ }^{\circ} \mathrm{C}$, washed with distilled water and allowed to dry before being stained with $0.4 \%$ SRB. The unbound SRB was washed with $0.1 \%$ acetic acid and the plates allowed to air dry. Bound SRB was dissolved in $10 \mathrm{mmol} / \mathrm{Tris} \mathrm{pH} 10.5$ and absorbances read at $495 \mathrm{~nm}$.

Caspase inhibition assay. GBM cell lines were seeded at a density of $1.5 \times 10^{5}$ cells per well in 6-well tissue culture-treated plates. Once cells had attached, they were then treated with or without $50 \mu \mathrm{M}$ Z-VAD-fmk (Bachem, Switzerland) for $24 \mathrm{~h}$ in order to inhibit caspase activity. Wells were then treated in the following manner: control (untreated $-/+$ Z-VAD; citrulline $1 \mathrm{mM}-/+$ Z-VAD; ADI-PEG20 $(0.1 \mu \mathrm{g} / \mathrm{ml}-/+$ Z-VAD). Cells were then left for $48 \mathrm{~h}$ under standard culture conditions and harvested for assessment of caspase and noncaspase-mediated cell death. Floating and attached cells (removed by trypsinisation) were then processed for flow cytometric analysis using the Annexin V-FITC Apoptosis Detection kit (Calbiochem, Nottingham, UK). Flow cytometric analysis was carried out using a Coulter EPICS flow cytometer (Beckman Coulter (UK) Ltd, High Wycombe, UK) with FL1 for FITC-annexin staining and FL3 for propidium iodide staining. Samples were analysed in duplicate and the experiments were set up on three separate occasions.

Inhibition of autophagy. Cells were treated with $10 \mu \mathrm{M} \mathrm{CQ}, 1 \mu \mathrm{g} / \mathrm{ml}$ ADIPEG20 or both for 24,48 and $72 \mathrm{~h}$. Cells were harvested, washed in PBS and resuspended in $500 \mathrm{ml} \mathrm{PBS}$ and fixed by the addition of $2 \mathrm{ml}$ of cold $90 \%$ methanol for at least $2 \mathrm{~h}$. After fixing, cells were washed twice in PBS and then re-suspended in $300 \mu \mathrm{l}$ of PBS containing $1 \mu \mathrm{g} / \mathrm{ml}$ propidium iodide and $10 \mathrm{mg} / \mathrm{ml}$ RNaseA and then analysed by flow cytometry for sub-G1 population.

ASS1 knockdown. Short hairpin RNA (shRNA) sequences targeting ASS1 were cloned into the pSilencer 4.1-CMVpuro vector (Ambion, Life Technologies Ltd). Sequences were designed by Ambion and supplied by Invitrogen (Supplementary Table S1). Control vector expressing an shRNA sequence that does not target any known human gene was used as a negative control in experiments. T98G, U118 and 42MG cells were transfected with shRNAexpressing vectors using Lipofectamine 1000 (Invitrogen). Stable pooled populations of transfectants were obtained following selection with $1 \mu \mathrm{g} / \mathrm{ml}$ puromycin for 3-4 weeks.

Generation of stable GFP-LC3 cells. LN229 cells were transfected with GFP-LC3 (CBA-401) expression vector or empty vector (Cambridge Bioscience, Cambridge, UK). Stable pools of transfectants were obtained following selection with $800 \mu \mathrm{g} / \mathrm{ml}$ of G418 for 3-4 weeks. These were expanded and stored in LN2 until required.

Flow cytometry for CD133 expression. For analysis of CD133 expression and cell sorting by flow cytometry, live cells were trypsinised, washed with cold PBS/1\%FCS (wash buffer) and re-suspended at a density of $1 \times$ $10^{6} \mathrm{cell} / \mathrm{s} / \mathrm{ml}$ in wash buffer. In all, $1 \times 10^{5}$ cells were incubated with $100 \mu \mathrm{l}$ antiCD133 antibody or mouse IgG1 isotype matched control (Miltenyl Biotech Ltd (Surrey, UK), diluted $1: 100$ in wash buffer) for $1 \mathrm{~h}$ at $4{ }^{\circ} \mathrm{C}$. After the incubation period, cells were washed twice in wash buffer incubated with $100 \mu$ l goat-antimouse FITC (Sigma-Aldrich) diluted 1:100 in wash buffer. Finally cells were washed twice in wash buffer and re-suspended in $500 \mu \mathrm{l}$ PBS/FCS before being analysed by flow cytometry and sorted for CD133-positive and -negative cells.

Statistics. Kaplan-Meier curves were used to estimate the probabilities of survival and time to progression and the log-rank test to assess the statistical significance of differences in event rates using Prism 5 (GraphPad software, Inc.,La Jolla, CA, USA).

Written informed consent was received from the participants before inclusion in the study.

\section{Conflict of Interest}

The authors declare no conflict of interest.

Acknowledgements. This work was supported by the Brain Tumour Research Campaign (BTRC) charity.

1. Gurney JG, Kadan-Lottick N. Brain and other central nervous system tumors: rates, trends, and epidemiology. Curr Opin Oncol 2001; 13: 160-166.

2. Preusser M, de Ribaupierre S, Wöhrer A, Erridge SC, Hegi M, Weller M et al. Current concepts and management of glioblastoma. Ann Neurol 2011; 70: 9-21.

3. Clarke J, Butowski N, Chang S. Recent advances in therapy for glioblastoma. Arch Neurol 2010; 67: 279-283.

4. Ziegler DS, Kung AL, Kieran MW. Anti-apoptosis mechanisms in malignant gliomas. J Clin Oncol 2008; 26: 493-500.

5. Ziegler DS, Wright RD, Kesari S, Lemieux ME, Tran MA, Jain M et al. Resistance of human glioblastoma multiforme cells to growth factor inhibitors is overcome by blockade of inhibitor of apoptosis proteins. J Clin Invest 2008; 118: 3109-3122.

6. Liu Q, Nguyen DH, Dong Q, Shitaku P, Chung K, Liu OY et al. Molecular properties of $\mathrm{CD}_{133^{+}}$glioblastoma stem cells derived from treatment-refractory recurrent brain tumors. J Neurooncol 2009; 94: 1-19.

7. Kögel D, Fulda S, Mittelbronn M. Therapeutic exploitation of apoptosis and autophagy for glioblastoma. Anticancer Agents Med Chem 2010; 10: 438-449.

8. Lefranc F, Facchini V, Kiss R. Proautophagic drugs: a novel means to combat apoptosisresistant cancers, with a special emphasis on glioblastomas. Oncologist 2007; 12 : 1395-1403.

9. Lefranc F, Kiss R. Autophagy, the Trojan horse to combat glioblastomas. Neurosurg Focus 2006; 20: E7.

10. Kanzawa $\mathrm{T}$, Germano IM, Komata $\mathrm{T}$, Ito $\mathrm{H}$, Kondo $\mathrm{Y}$, Kondo $\mathrm{S}$. Role of autophagy in temozolomide-induced cytotoxicity for malignant glioma cells. Cell Death Differ 2004; 11: 448-457.

11. Clarke PG. Developmental cell death: morphological diversity and multiple mechanisms. Anat Embryol (Berl) 1990; 181: 195-213.

12. Yang Z, Klionsky DJ. Mammalian autophagy: core molecular machinery and signaling regulation. Curr Opin Cell Biol 2010; 22: 124-131.

13. Haines RJ, Pendleton LC, Eichler DC. Argininosuccinate synthase: at the center of arginine metabolism. Int J Biochem Mol Biol 2011; 2: 8-23.

14. Gong H, Zölzer F, von Recklinghausen G, Havers W, Schweigerer L. Arginine deiminase inhibits proliferation of human leukemia cells more potently than asparaginase by inducing cell cycle arrest and apoptosis. Leukemia 2000; 14: 826-829.

15. Ensor CM, Holtsberg FW, Bomalaski JS, Clark MA. Pegylated arginine deiminase (ADI-SS PEG20,000 mw) inhibits human melanomas and hepatocellular carcinomas in vitro and in vivo. Cancer Res 2002; 62: 5443-5450.

16. Yoon C-Y, Shim Y-J, Kim E-H, Lee JH, Won NH, Kim JH et al. Renal cell carcinoma does not express argininosuccinate synthetase and is highly sensitive to arginine deprivation via arginine deiminase. Int J Cancer 2007; 120: 897-905.

17. Bowles TL, Kim R, Galante J, Parsons CM, Virudachalam S, Kung HJ et al. Pancreatic cancer cell lines deficient in argininosuccinate synthetase are sensitive to arginine deprivation by arginine deiminase. Int J Cancer 2008; 123: 1950-1955.

18. Kim RH, Coates JM, Bowles TL, McNerney GP, Sutcliffe J, Jung JU et al. Arginine deiminase as a novel therapy for prostate cancer induces autophagy and caspaseindependent apoptosis. Cancer Res 2009; 69: 700-708.

19. Delage B, Fennell DA, Nicholson L, McNeish I, Lemoine NR, Crook T et al. Arginine deprivation and argininosuccinate synthetase expression in the treatment of cancer. Int $J$ Cancer 2010; 126: 2762-2772.

20. Kelly MP, Jungbluth AA, Wu BW, Bomalaski J, Old LJ, Ritter G. Arginine deiminase PEG20 inhibits growth of small cell lung cancers lacking expression of argininosuccinate synthetase. Br J Cancer 2011; 106: 324-332.

21. Nicholson LJ, Smith PR, Hiller L, Szlosarek PW, Kimberley C, Sehouli J et al. Epigenetic silencing of argininosuccinate synthetase confers resistance to platinum-induced cell death but collateral sensitivity to arginine auxotrophy in ovarian cancer. Int J Cancer 2009; 125: 1454-1463.

22. Savaraj N, You M, Wu C, Wangpaichitr M, Kuo MT, Feun LG. Arginine deprivation, autophagy, apoptosis (AAA) for the treatment of melanoma. Curr Mol Med 2010; 10: 405-412.

23. Wheatley DN. Arginine deprivation and metabolomics: important aspects of intermediary metabolism in relation to the differential sensitivity of normal and tumour cells. Semin Cancer Biol 2005; 15: 247-253.

24. Bizzoco E, Faussone-Pellegrini MS, Vannucchi MG. Activated microglia cells express argininosuccinate synthetase and argininosuccinate lyase in the rat brain after transient ischemia. Exp Neurol 2007; 208: 100-109.

25. Etcheverry A, Aubry M, de Tayrac M, Vauleon E, Boniface R, Guenot F et al. DNA methylation in glioblastoma: impact on gene expression and clinical outcome. BMC Genomics 2010; 11: 701 .

26. Stupp R, Hegi ME, Mason WP, van den Bent MJ, Taphoorn MJ, Janzer RC et al. Effects of radiotherapy with concomitant and adjuvant temozolomide versus radiotherapy alone on 
survival in glioblastoma in a randomised phase III study: 5-year analysis of the EORTC NCIC trial. Lancet Oncol 2009; 10: 459-466.

27. Weller M, Stupp R, Reifenberger G, Brandes AA, van den Bent MJ, Wick W et al. MGMT promoter methylation in malignant gliomas: ready for personalized medicine? Nat Rev Neurol 2010; 6: 39-51.

28. Fafournoux $\mathrm{P}$, Bruhat $\mathrm{A}$, Jousse $\mathrm{C}$. Amino acid regulation of gene expression. Biochem $J$ 2000; 351(Pt 1): 1-12.

29. Chaveroux C, Lambert-Langlais S, Cherasse Y, Averous J, Parry L, Carraro V et al. Molecular mechanisms involved in the adaptation to amino acid limitation in mammals. Biochimie 2010; 92: 736-745.

30. Briceño $E$, Reyes $S$, Sotelo J. Therapy of glioblastoma multiforme improved by the antimutagenic chloroquine. Neurosurg Focus 2003; 14: e3.

31. Briceño E, Calderon A, Sotelo J. Institutional experience with chloroquine as an adjuvant to the therapy for glioblastoma multiforme. Surg Neurol 2007; 67: 388-391.
32. Parsons DW, Jones $S$, Zhang $X$, Lin JC, Leary RJ, Angenendt $P$ et al. An integrated genomic analysis of human glioblastoma multiforme. Science 2008; 321: 1807-1812.

33. Geng Y, Kohli L, Klocke BJ, Roth KA. Chloroquine-induced autophagic vacuole accumulation and cell death in glioma cells is p53 independent. Neuro Oncol 2010; 12: 473-481.

Cell Death and Disease is an open-access journal published by Nature Publishing Group. This work is licensed under the Creative Commons Attribution-NonCommercial-No Derivative Works 3.0 Unported License. To view a copy of this license, visit http://creativecommons.org/licenses/by-nc-nd/3.0/

Supplementary Information accompanies the paper on Cell Death and Disease website (http://www.nature.com/cddis) 